\title{
Review of Tool-Tissue Interaction Models for Robotic Surgery Applications
}

\author{
Árpád Takács, Sándor Jordán, Radu-Emil Precup*, Levente Kovács, József Tar, Imre Rudas and Tamás Haidegger \\ Antal Bejczy Center for Intelligent Robotics \\ Óbuda University, Budapest, Hungary \\ \{arpad.takacs, sandor.jordan, tamas.haidegger\}@irob.uni-obuda.hu, \{kovacs.levente, tar.jozsef\}@nik.uni-obuda.hu, \\ rudas@uni-obuda.hu \\ *Dept. of Automation and Applied Informatics, Politehnica University of Timisoara, Romania; radu.precup@aut.upt.ro
}

\begin{abstract}
Besides the motion control issues and problems arising in general robotic applications, control engineers frequently encounter difficulties in designing robotic surgery systems due to the complex environmental constraints present. One of the most challenging problems is caused by the unique behavior of soft tissues under manipulations such as grabbing, cutting and indentation. The appropriate modeling of the interaction between the tools held by the robotic arm and tissues is essential for precise control, yet no generally applicable, universal model has been developed so far. This paper gives an overview of the existing tool-tissue interaction models and possible approaches to the parameterization problem, listing and reviewing several models found in the literature. We reviewed the problem within the frames of a theoretical master-slave teleoperation surgical robot, where exact mathematical model of the soft tissue is essential for effective control over a time-delayed communication channel.
\end{abstract}

Keywords-Telesurgery; Time delay; Tissue modeling; Tooltissue interactions.

\section{INTRODUCTION}

In the early 1970s, NASA created the first concept of long distance surgical procedures that would be performed or supported by robots [1]. According to the initial concept the primary use of these robots would be in the Earth-space distance, supporting astronauts on space missions. In the past decades, instead of telesurgery in space, focus has been drawn on teleoperation over shorter distances. In 2001, the first successful intercontinental teleoperation was carried out [2] and the concept appeared in military applications such as the TRAUMA POD project, demonstrating a technology serving as a remote tool and assistant for the medical crews in war zones [3]. Although many applications and concepts were developed, many issues remained unsolved, due to the great number of components in teleoperation systems and the lack of specific knowledge about system behavior. An overview of the major modeling issues in robotic telesurgery application was presented in [4], providing details of questions regarding the three major components of teleoperation systems: the master side, the slave side and the communication channel. Proper modeling and control of all components still remains an important research topic. In this paper, we focus on questions regarding of the slave side modeling, concentrating the tissue behavior under robotic manipulation.

\section{COMPONENTS OF TELEOPERATION SYSTEMS}

From the modeling and control point of view, surgical robots and teleoperation systems consist of three major components: the master device, the slave device and the communication system (Fig. 1). Tool-tissue interaction models are represented by the slave side, categorized as a subcomponent. It is clear that in the case of a fully operational telesurgical application, all components must be assessed in terms of mathematical modeling, both individually and as a whole, thus the problem complexity may reach exceptionally high levels.

\section{A. Communication system}

The communication system may be modeled as the assembly of three major parts: the transmitter, the receiver and the communication medium. Signal quality and the latency levels are determined by these components. However, one must also deal with package loss and compatibility issues. The most commonly addressed is the time delay arising due to the long distance travel of information. The deteriorating effects of time delay could be reduced by using latency-tolerant control methods, or, in some cases, utilizing predictive controllers tuned to the master and slave systems [5]. The simplest way to model time delay in frequency domain is given by the formula [6]:

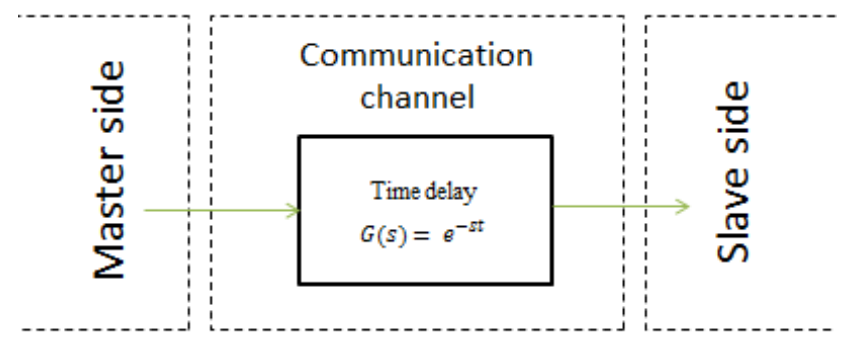

Figure 1. Block diagram of time delay model used in robotic surgery modeling [4]. 


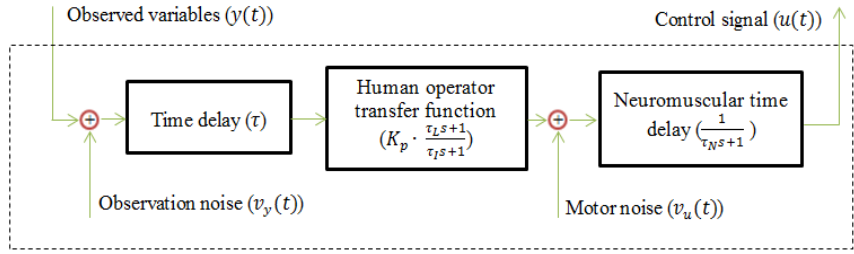

Figure 2. Multivariable model of a human, operating as a controller.

$$
y(s)=G(s) u(s)=e^{-s \tau} u(s),
$$

where the signal delay can be modeled by the $G(s)=e^{-s \tau}$ transfer function, and $\tau$ is the time delay. Accepting zero initial conditions, in time domain, (1) can be written as follows:

$$
y(t)=u(t-\tau) .
$$

The most simple concept of time delay is illustrated in Fig.1.

\section{B. Master model}

The human operator or the substituting automatic control device is located at the master side. It can be expanded with the surgical staff that is capable of sending control signals to the slave side. The crossover model was developed in the 1960s and has been widely used since then to create a mathematical model of the controlling human operator, although initially it was designed to model fighter pilots [7]. The crossover model is based on the highly non-linear and time-dependent response of the human body. However, it does not take other human factors such as tiredness and stress into account. It is widely used due to its quasi-linear appearance. Another possible approach to human operator modeling was introduced by Ornstein [8], which is a more general, parametric, complex description of master side behavior, also applicable for pursuit-type manual tracking tasks [9]. A general diagram of the human operator is shown in Fig. 2.

\section{Slave model}

In order to reduce the possible disturbance of latency that could not be avoided, modeling of the slave side is essential to increase the robustness and reliability of the process model. More precisely, the appropriate choice of control methods like MPC (Model Predictive Control) empowered with the detailed model of kinematic [10], [11], and tool-tissue interaction behavior at the end of the robot arm can largely reduce the effect of latency. One of the most challenging issues is the acquisition of the right model of specimens, or in case of surgical robotics, the model of tissues.

There are significant differences between addressing problems involving soft tissues and hard tissues. While this paper exclusively focuses on soft tissue manipulation, where the right modeling of the tissues is essential, it is important to mention that modeling of hard tissues and their interaction with the robotic arm, such as bone drilling, carries many issues closely related to the field of machining technology studies.

In general, these tissues are highly nonlinear, inhomogeneous, anisotropic, viscoelastic materials and the modeling is of high importance not only in robot control but also in the use of surgical simulators.

\section{TOOL-TISSUE INTERATCION MODELS}

A comprehensive study about the existing soft tissue models used in most MIS applications and virtual surgical simulators was presented by Famaey and Sloten [12], introducing three major categories of deformation models: heuristic models, continuum-mechanics models and hybrid models. The complexity of each model mentioned above varies on a wide scale, although it is commonly accepted that approaches based on continuum-mechanics provide a more realistic response, but require significantly higher computational capacity. Analytical solution to the used mathematical laws generally does not exist. On the contrary, heuristic models that consist of lumped, linear mass-springdamper elements, sometimes also called mechanical models can be used for describing simple surgical tasks like needle insertion. The derived equations can usually be solved analytically.

In Fig. 3, the most commonly used mechanical models are shown for soft tissue modeling. Besides their complexity and the ordering of the components, each model represents a typical behavior and thus a physical meaning that is rarely discussed in literature.

Pure elastic approach is omitted in the listing above. However, in some cases when the applied strain is less than $1 \%$, the use of this simple model can be justified. The simplest approaches to describe viscoelasticity are the Maxwell and the Voigt models. In the Maxwell model, a serial connection of a spring and a damper present the process of total stress relaxation, providing a non-realistic response of the tissue. In this case, an infinitely slow interaction would cause no resistance from the specimen side. On the contrary, the Voigt model preserves the elasticity of the tissue. However, its drawback is the lack of realistic modeling of rapid interactions, thus a step-input would cause an infinitely large reaction force. It is the Kelvin model that provides the most realistic description of tissues of the three, compensating for the deficiencies of Maxwell and Voigt models. The unknown parameters of the spring and damper components are usually obtained from specimen-indentation tests by curve fitting and are used for further calculations.

One of the greatest advantages of this approach is the modularity and the possible existence of analytical solution. The models can be combined up to a desired complexity level,

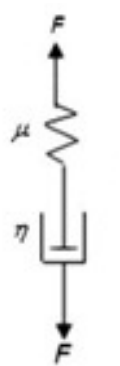

(a)

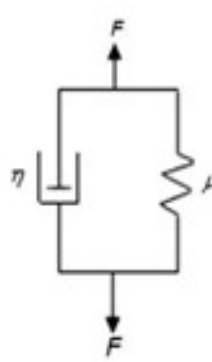

(b)

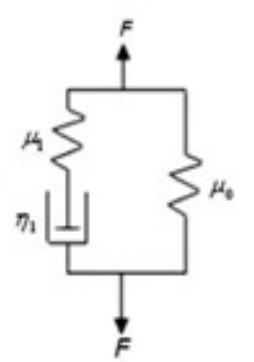

(c)
Figure.3. The most commonly used mechanical models of viscoelasticity: a) Maxwell model b) Voigt model c) Kelvin model [13]. 
introducing more parameters, thus bringing the system response closer to realistic. The generalized Maxwell model, also called the Maxwell-Wiechert model, is the most widely used generalized mechanical approach.

While the modeling of soft tissue behavior has been in the focus of research for long, the challenging field of gaining information about the interactions of the robot arm and the tissue has only reached popularity recently. Among the arising issues it is important to mention the problem of force feedback, the modeling of tools and the interaction with organs itself. A comprehensive review on current tool-tissue interaction models was carried out in [14], providing a survey on research focusing on interactions described by models, following the principles of continuum mechanics and finite element methods. In this work, we extend the area of interest to models of telesurgical applications, without strict boundaries of categories, giving an overview of model properties.

In [15], a simple 1-Degree-of-Freedom (DoF) model of a rigid master and flexible slave connection was introduced. Here, the problem of tool flexibility is addressed as one of the greatest issues in the case of tool tissue interactions, since the force sensing can only be applied at the fixed end of the tool and its deflection can only be estimated. Besides tool flexibility, the compliant parameters of the robotic arm and the tissue model are also important and significant parts of the tool tissue interaction system. Other extensions of the model exist for rigid slave, flexible joint and flexible master descriptions, the complexity of the model of the whole system can be extremely high. Great advantage of this approach is that not only the tool flexibility but the whole transparency of the system is addressed. It is important to mention though, that no detailed tissue modeling is provided, the use of rigid specimen model indicates that this approach is rather focusing on teleoperation.

Basdogan et al [16] addressed the importance of tool tissue interaction modeling in medical training through simulation in virtual reality, focusing on issues in haptics in minimally invasive surgery. When working with soft tissues, the elastic behavior of the tool can usually be omitted, using rigid models of surgical accessories. In their work, they introduced two new approaches to tissue modeling: the mesh-based FEA model, using modal analysis and the real-time meshless method of finite spheres. In the virtual environment, collision detection and perception of multiple tissue layers was created, accompanied with force and torque feedback to user's hand. This feature is supported by force and position sensors mounted on the tool, which is held by the user instead of a robotic arm. The complexity of the above mentioned methods is in connection with the required computational effort. In simple problems the use of the method of finite spheres is suggested. Another approach to meshless methods was introduced by Bao et al., where several layers were used as the model of the soft tissue, their interaction modeled with a heuristic Kelvin model [17]. Modeling of two important viscoelastic properties, the creep and relaxation is possible with this new three-parameter viscoelastic model, improving the performance of conventional mass-spring-damper approaches.
Yamamoto suggested a method for the detection of lumps in organ tissues such as kidney, liver and heart [18]. The importance of this work was a comprehensive comparison of seven different tissue models used in point-to-point palpation. The aim of the tests and model validations was to create a graphical overlay system that stores data on palpation results, creating a color scale overlay on the actual tissue, processing the acquired data using several tissue models, with a single 1 DoF force sensor at the fixed end of the tool.

Yamamoto et al. also created an interpolable interface with haptic feedback and augmented visual feedback and performed palpation and surface detection tasks using visionbased forbidden-region virtual fixtures [19]. The tests were carried out on manufactured artificial tissues based on existing commercially available artificial prostate, using a complex, but-based on previous measurements - accurate HuntCrossley model. Position, velocity and force sensors were mounted on slave manipulator and the visual feedback to the human user was generated with a stereo-vision system.

When dealing with viscoelastic materials interacting with tools, coupled problems arise where additional mechanical models are required to describe the system response. It is important to mention that even when the best-suited mathematical models are employed, material properties (Young-modulus, Poisson-ratio, etc.) can only be estimated. Validation of their values requires circumstantial physical experiments. When using heuristic, mechanical tissue models, the acquisition of explicit, but general material properties are omitted. Instead of using tables and possible ranges of these properties, spring and damping coefficients must be obtained from measurements even when nothing else but the tool shape is changed. In their work, Leong et al. introduced and validated a mechanical model of liver tissue and its interaction with scalpel blade, creating a distributed model of mechanical viscoelastic elements [13]. With the serial connection of a Maxwell and Kelvin element they introduced the MaxwellKelvin viscoelastic body. The primary aim of this work was to account for the tissue surface deformation due to the extensive shape of the tool, validating with the cutting experiment where a 1 DoF force sensor was placed at the scalpel blade holder integrated with position measurement. Besides many constitutive ideas, a great number of deficiencies can be found in the model that still needs to be improved, including mathematical errors in modeling, contradictions in the measurement result evaluation, inappropriate use of Laplace transformation and the pertinence of experimental results.

Liu et al. introduced a method for force control for roboticassisted surgery on beating heart, thus applying motion compensation for the periodic motion of the organ [20]. By installing a force sensor at the end of the instrument and tracking the $3 \mathrm{D}$ motion of the beating heart, they compared four different models from the viewpoint of tracking performance of the desired force. Besides the conventional viscoelastic models, a fourth, fractional derivative model of viscosity was examined. One of the relevant results of this experiment was to underline the importance of the right choice of tissue model. 


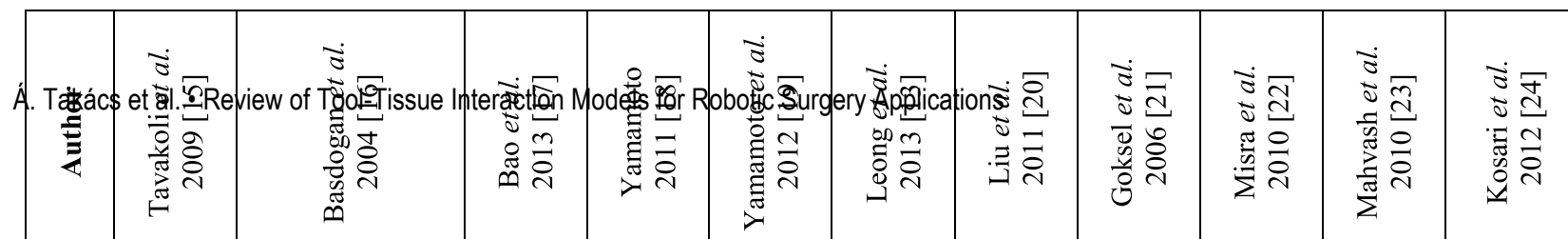

\begin{tabular}{|c|c|c|c|c|c|c|c|c|c|c|c|c|}
\hline 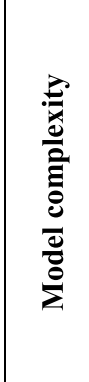 & 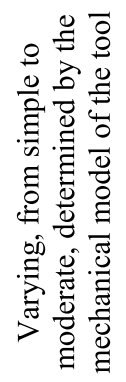 & 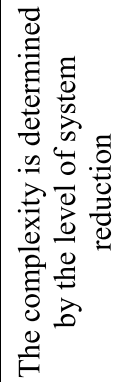 & 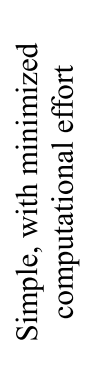 & 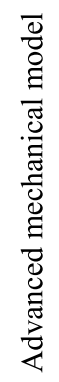 & 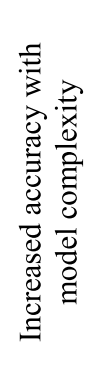 & 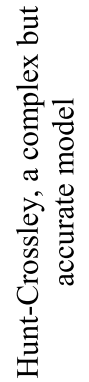 & 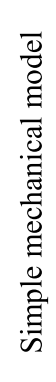 & 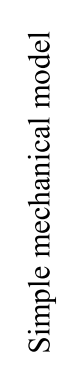 & 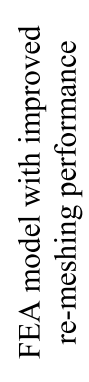 & 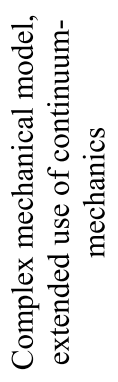 & 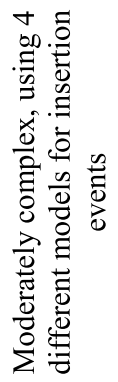 & 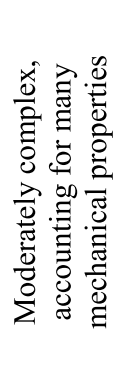 \\
\hline
\end{tabular}

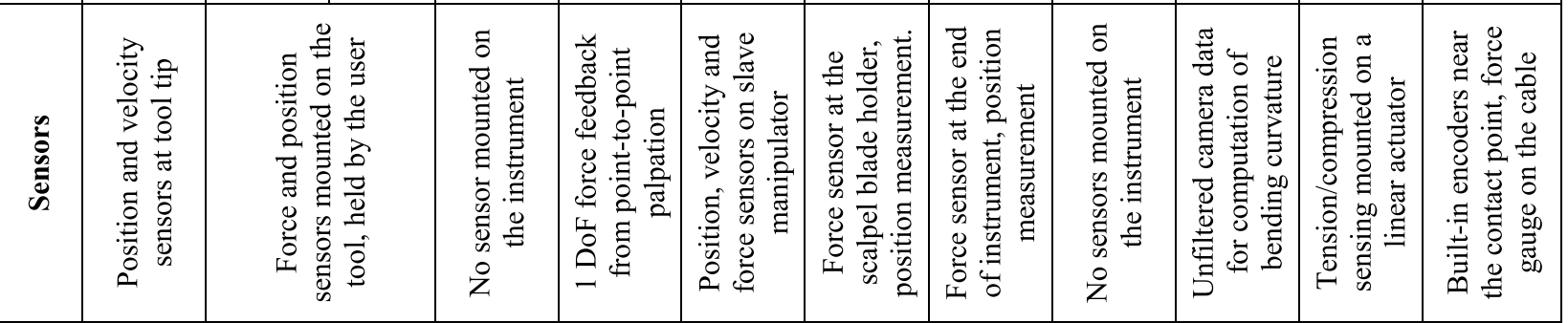

\begin{tabular}{|c|c|c|c|c|c|c|c|c|c|c|}
\hline 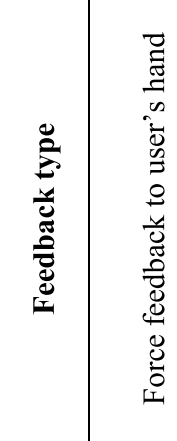 & 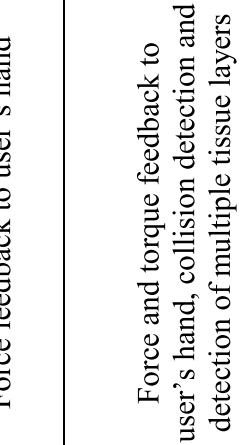 & 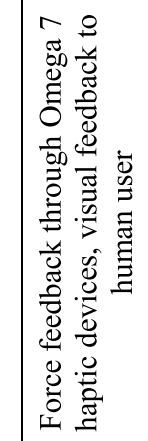 & 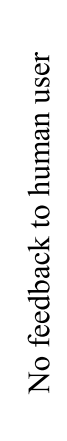 & 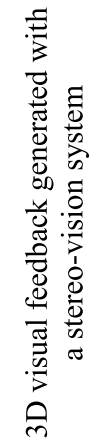 & 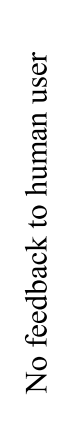 & 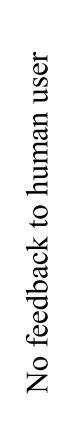 & 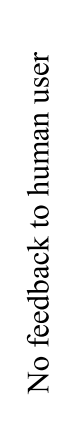 & 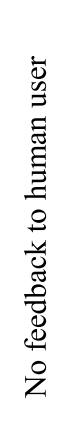 & 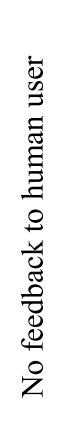 & 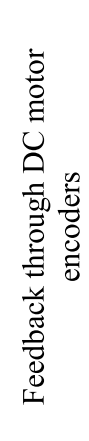 \\
\hline
\end{tabular}

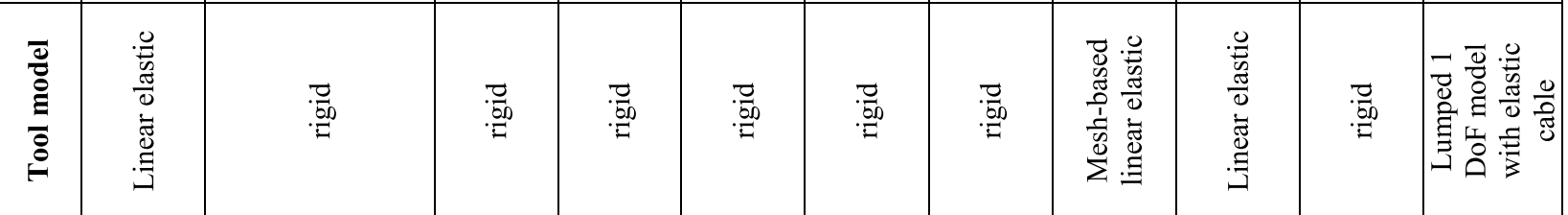

\begin{tabular}{|c|c|c|c|c|c|c|c|c|c|c|c|c|}
\hline 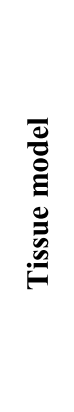 & $\frac{\vec{D}}{\overrightarrow{0}}$ & 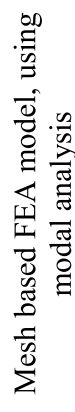 & 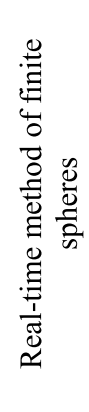 & 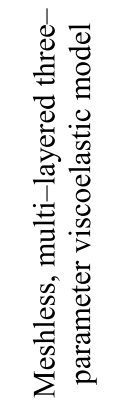 & 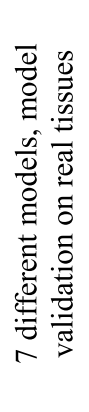 & 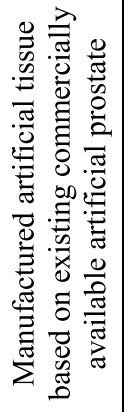 & 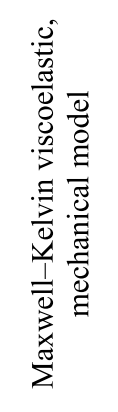 & 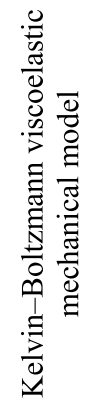 & 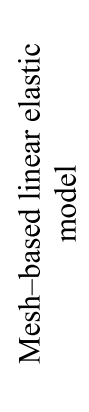 & 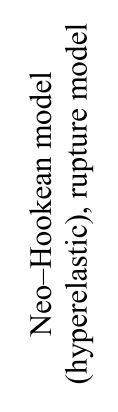 & 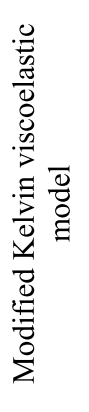 & 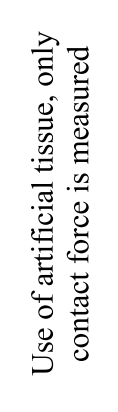 \\
\hline 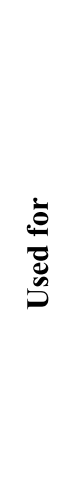 & 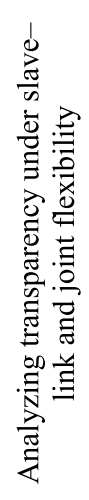 & & & 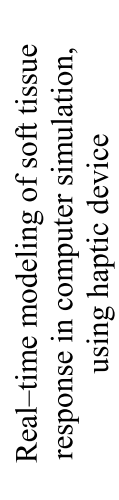 & 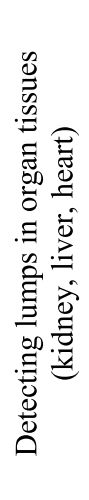 & 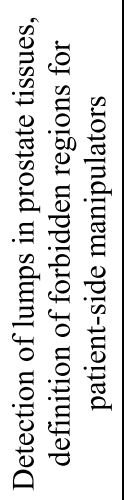 & 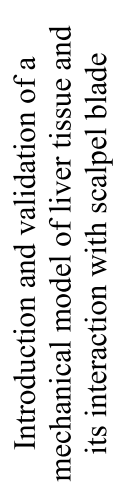 & 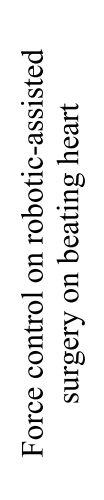 & 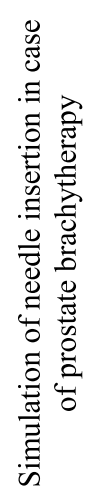 & 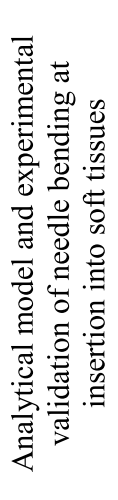 & 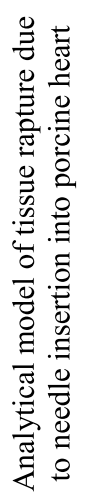 & 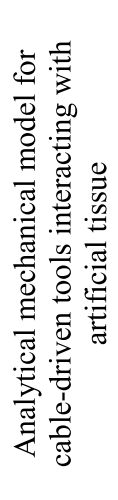 \\
\hline 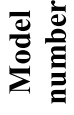 & - & & & $m$ & f & 342 & 6 & $r$ & $\infty$ & $a$ & $=$ & \\
\hline
\end{tabular}


In the past years, much focus has been drawn on needle insertion modeling. Due to the simplicity of the tool geometry, needle insertion problems were much discussed using Finite Element modeling. Finite Element Method is a widely used approach for tool tissue interaction modeling, where commercially available FEA software packages are used to aid and simulate the operation area. The great many built inmechanical models can provide incredibly accurate and realistic solution for simulation. One of the largest drawbacks of this method is the sensitivity of computational time length with respect to the parameters used in FE simulations. These parameters are determined solely by the user, including spatial and time resolutions, thus many simulations need to be carried out on the same model to achieve the desired level of reliability.

Goksel et al. introduced a novel technique to use real-time remeshing in the case of FEA modeling [21]. A mesh-based linear elastic model of both the needle and tissue was used, applying remeshing in order to compensate organ shift due to the invasiveness. The importance of the model is that both tool and tissue deformation were accounted for, although the motion models were the simplest possible in 3D.

Continuum mechanics also provides numerous models that can be used for modeling organ and tissue deformations and kinetics. Approaches using linear and nonlinear models of elasticity are widely used in practice. Linear models have limited usability despite the many advantages they carry (simplicity, easy-calculation and small requirements on computational capacity) due to inhomogeneous, anisotropic, non-linear characteristics of tissues and large relative deformations, strains. However, nonlinear models in continuum mechanics lead to moderately complex models even in simple surgical tasks. Misra et al. introduced a detailed complex mechanical model of continuum mechanics for the analytical modeling and experimental validation of needle bending at insertion into soft tissues [22]. A hyperelastic Neo-Hookean rupture model was used to describe the material properties and behavior of the soft-tissue simulant (gel), assuming linear elasticity in case of the needle. Experiments were carried out using different bevel-tipped needles and the needle bending curvature was validated using an unfiltered camera data. The importance of the work lays in the area of needle insertion path planning.

In the area of tool-tissue interaction research, one might be interested in rapture modeling. While most of the existing mechanical models assume reversible tissue deformation, even in the case of minimal invasive surgery (MIS), tissue rupture cannot be avoided. Mahvash and Dupon [23] developed an analytical model of tissue rapture during needle insertion, focusing on the calculation of required insertion force. The great advantage of this model is that despite the complex mechanical structure, the insertion events are divided into four different models, decomposing the process into moderately complex parts. Tissue modeling was aided with a modified Kelvin model, making the parameters of the linear components dependent of the deformation rate. The analytical model validated the experiments showing that the required insertion force is inversely proportional to the insertion speed.
It is important to mention models that are not directly describing insertion and cutting problems, but are rather used for investigating interaction of cable-driven manipulators controlled by human operators, acting on soft tissues. Kosari et al. introduced an adaptive parameter estimation and MPC method on cable-driven surgical manipulators, developing a 1 DoF mechanical model, concentrating on the problem of trajectory tracking [24]. Therefore, instead of the estimation of tissue reaction forces, focus was drawn to the response of the cable-driven manipulator in order to create a realistic force feedback to human user. The moderately complex model accounts for numerous mechanical properties and solves an optimal control problem for automating tissue compression.

The proper modeling of tool-tissue interactions is a relevant topic in standardization methods. With the help of initial calculations and simulations, efficient control methods can be chosen to avoid undesired pain and injury levels. Pain and injury onset levels for static contact force and peak pressure values has been deeply researched and standardized in the literature [25].

A summary of the above mentioned models can be seen in Table 1, discussing details of tool and tissue models, feedback modeling, proposed or used sensor assembly and level of complexity.

\section{CONCLUSION}

Telesurgical applications have gained much attention in the past years, boosted by rapidly developing robotics technology and advancements in biological and surgical fields. The right choice of models is essential for further development all for the basic components of teleoperation systems and for tool-tissue interactions. In this work, we presented some of the most significant approaches in the field of tool-tissue interactions. However, it is important to mention that extending these models with some of the major issues of the rest of the components such as latency, haptics or the model of human operator, fundamentally new approaches can be generated, requiring an extensive knowledge in all of the connected fields of study.

As a result of our research, great potential has been found in some of the investigated models that can be used as a pillar of the forthcoming work. Particularly, the idea of distributed mechanical models introduced by Leong et al. [13] can be extended and corrected, then later to be validated by experimental results. From the force control point of view, the work of Liu et al. [20] proves to be very useful to understand tool-tissue modeling and force tracking under organ motion. Finally, on the tool side, many useful ideas and guidelines to modeling was given by Kosari et al. [24].

It is common sense to state that the use of simple models is suggested in order to keep the overall rank of the system at a low level, taking the general rule of modeling into account: if a model is too simple, the behavior of the real system will differ from the simulated one. On the other hand, if the model is too complex, one may not be able to solve the problem. 


\section{ACKNOWLEDGMENT}

Tamás Haidegger and Levente Kovács are Bolyai Fellows of the Hungarian Academy of Sciences.

\section{REFERENCES}

[1] T. Haidegger, "Surgical Robot Prototyping-System Development, Assessment and Clearance," ch. 10 in T. Sobh and X. Xiong, Eds. Prototyping of Robotic Systems: Applications of Design and Implementation, IGI Book, Bridgeport, CT, pp. 288-326, 2012.

[2] T. Haidegger, J. Sandor and Z. Benyo, "Surgery in Space: the Future of Robotic Telesurgery," Surgical Endoscopy, vol. 25, no. 3, pp. 681-690, 2011.

[3] P. Garcia, J. Rosen, C. Kapoor, M. Noakes, G. Elbert, M. Treat, T. Ganous, M. Hanson, J. Manak, C. Hasser, D. Rohler, and R. Satava, "Trauma pod: a semi-automated telerobotic surgical system," J. Medicine, vol. 5, no. 2, pp. 136-146, 2009.

[4] S. Jordan, A. Takacs, I. Rudas and T. Haidegger, "Modelling and Control Framework for Robotic Telesurgery," in Proc. $3^{\text {rd }}$ Joint Workshop on New Technologies for Computer/Robot Assisted Surgery (CRAS), Verona, 2013.

[5] R.-E. Precup, T. Haidegger, L. Kovacs, A. S. Paul, S. Preitl and Z. Benyo, "Fuzzy Control Solution for Telesurgical Applications," Applied and Computational Mathematics. An International Journal (ACM), vol. 11, no. 3, pp. 378-397, 2012.

[6] K. J. Åström and R. M. Murray, Analysis and Design of Feedback Systems, chapter 6. Preprint, 2004, pp. 139-156

[7] D. McRuer and H. Jex, "A Review of Quasi-Linear Pilot Models," IEEE Transactions on Human Factors in Electronics, vol. 8, no. 3, pp. 231249, Sep. 1967.

[8] G. Ornstein, "The Automatic Analog Determination of Human Transfer Function Coefficients.” Med. Electron. Bio. Eng., vol. 1, no. 3, 1963.

[9] D. Kleinman, S. Baron and W. Levison, "An optimal control model of human response part I: Theory and validation", Automatica, vol. 6, no. 3, pp. 357-369, 1970.

[10] L. W. Sun, F. Van Meer, Y. Bailly and C. K. Yeung, "Design and development of a Da Vinci surgical system simulator," in Proc. IEEE Intl. Conf. on Mechatronics and Automation (ICMA), pp. 1050-1055, 2007.

[11] A.A. Syed, X. G. Duan, X. Kong, M. Li, Y. Wang and Q. Huang, "6DOF Maxillofacial Surgical Robotic Manipulator Controlled By Haptic Device," in Proc. $9^{\text {th }}$ International Conference on Ubiquitous Robots and Ambient Intelligence (URAI 2012), Daejeon, pp. 71-74, 2012.

[12] N. Famaey and J. Vander Sloten, "Soft tissue modelling for applications in virtual surgery and surgical robotics", in Computer Methods in
Biomechanics and Biomedical Engineering, vol. 11, no. 4, pp 351-366, 2008.

[13] F. Leong, W. Huang and C. Chui, "Modelling and analysis of coagulated liver tissue and its interaction with a scalpel blade," Medical and Bioogicall Engineering Computing, vol. 51, pp. 687-695, 2013.

[14] S. Misra, K. T. Ramesh and Allison M. Okamura, "Modeling of ToolTissue Interactions for Computer-Based Surgical Simulation: A Literature Review," Presence: Teleoperators and Virtual Environments, vol. 17, no. 5, pp. 463-491, 2008.

[15] M. Tavakoli and Robert D. Howe, "Haptic Effects of Surgical Teleoperator Flexibility," The International Journal of Robotics Research, vol. 28, no. 10, pp.1289-1302, 2009.

[16] C. Basdogan, S. De, J. Kim et al., "Haptics in Minimally Invasive Surgical Simulation and Training", IEEE Computer Graphics and Applications, vol. 24, no. 2, pp. 56-64, 2004.

[17] Y. Bao, D. Wu, Z. Yan and Z. Du, "A New Hybrid Viscoelastic Soft Tissue Model based on Meshless Method for Haptic Surgical Simulation". The Open Biomedical Engineering Journal, vol. 7, pp. 116-124, 2013.

[18] T. Yamamoto, "Applying Tissue Models in Teleoperated RobotAssisted Surgery," PhD dissertation, Johns Hopkins University, Baltimore, MD. January, 2011.

[19] T. Yamamoto, N. Abolhassani, S. Jung et al., "Augmented reality and haptic interfaces for robot-assisted surgery," The International Journal of Medical Robotics and Computer Assisted Surgery, vol. 8, pp. 45-56, 2012.

[20] C. Liu, P. Moreira, N. Zemiti and P. Poignet, "3D Force Control for Robotic-Assisted Beating Heart Surgery Based on Viscoelastic Tissue Model," in Proc. Int. Conf. of the IEEE Engineering in Medicine and Biology Society, pp. 7054-7058, 2011.

[21] O. Goksel, S. E. Salcudean and S. P. Dimaio, "3D simulation of needletissue interaction with application to prostate brachytherapy", Computer Aided Surgery, vol. 11, no. 6, pp. 279-288, 2006.

[22] S. Misra, K. B. Reed, B. W. Schafer et al., "Mechanics of Flexible Needles Robotically Steered through Soft Tissue", The International Journal of Robotics Research, vol.29, no.13, pp. 1640-1660, 2010.

[23] M Mahvash and P. E. Dupont, "Mechanics of Dynamic Needle Insertion into a Biological Material," IEEE Transactions on Biomedical Engineering, vol. 57, no. 4, pp. 934-943, 2010.

[24] S. N. Kosari, S. Ramadurai, H. J. Chizeck and B. Hannaford, "Robotic Compression of Soft Tissue," in Proc. IEEE International Conference on Robotics and Automation, St. Paul, 2012.

[25] Robots and robotic devices - Safety requirements for industrial robots - Collaborative operation, ISO/CD Standard 15066, 2013. 